\title{
Small eigenvalues of the Witten Laplacian acting on $p$-forms on a surface
}

\author{
D. Le Peutrec \\ Institut für Angewandte Mathematik, Universität Bonn, \\ Endenicher Allee 60, 53115 Bonn, Germany. \\ email: dorian.lepeutrec@uni-bonn.de
}

March 8, 2010

\begin{abstract}
In this article, we are interested in the exponentially small eigenvalues of the self adjoint realization of the semiclassical Witten Laplacian $\Delta_{f, h}^{(p)}$, in the general framework of $p$-forms, on a connected compact Riemannian manifold without boundary. Our purpose is to notice that the knowledge of (the asymptotic formulas for) the smallest non zero eigenvalues of the self adjoint realization of $\Delta_{f, h}^{(0)}$ (acting on functions), presented in $[\mathrm{HeKlNi}]$, essentially contains all the necessary information to the treatment of the case of oriented surfaces, for $p$-forms. The function $f$ is assumed to be a Morse function on $\Omega$.
\end{abstract}

MSC 2010: 58J37, 58J10, 81Q10, 58A10, 15A18.

Key words and phrases: Witten complex, exponentially small eigenvalues, differential p-forms on surfaces.

\section{Introduction and main result}

The study of the small eigenvalues of some self adjoint realization of the Witten Laplacian acting on functions (i.e. on 0-forms), $\Delta_{f, h}^{(0)}$, where $f$ is a Morse function, relies closely on the study of the metastability in reversible diffusion processes: the inverses of these small eigenvalues can indeed be interpreted as the mean exit times of local wells for a particle in a gradient field deriving from the function $f$. Similar problems were considered by many authors 
via a probabilistic approach in [FrWe], [HoKuSt], [Mic], and [Kol]. More recently, in the case of $\mathbb{R}^{n}$, accurate asymptotic forms of the exponentially small eigenvalues were obtained in $[\mathrm{BoEcGaKl}]$ and [BoGaKl]. These results were improved and extended to the cases of boundaryless compact manifolds in [HeKlNi] and of compact manifolds with boundaries for the Dirichlet and Neumann realizations of the Witten Laplacian in [HeNi] and [Lep1].

Nevertheless, the only case of the 0-forms has been fully treated. A natural question then remains the accurate study of the small eigenvalues of $\Delta_{f, h}^{(p)}$, the Witten Laplacian acting on $p$-forms, for general $p$-forms. Note also that, if the general case of $p$-forms has not been fully understood yet, the number of small eigenvalues of the self adjoint realizations of $\Delta_{f, h}^{(p)}$ is well known for every $p$ : in the case of a compact manifold without boundary, the number $m_{p}$ of eigenvalues of the self adjoint realization of the Witten Laplacian $\Delta_{f, h}^{(p)}$ in some interval $\left[0, C h^{\frac{3}{2}}\right]$ (for $h>0$ small enough) is the number of critical points of $f$ with index $p$ (see e.g. [Wit][Hen][HeSj4]).

In this connection, in the above references [BoEcGaKl, BoGaKl, HeKlNi, $\mathrm{HeNi}$, Lep1], the accurate computation of the $m_{0}$ smallest eigenvalues of $\Delta_{f, h}^{(0)}$ is done after coupling the $m_{0}$ local minima with some relevant critical points with index 1 . This coupling relies on some topological aspects. The constant $C$ in the exponential terms $e^{-\frac{C}{h}}$ of these small eigenvalues are then on the form $f\left(U^{1}\right)-f\left(U^{0}\right)$, where $U^{0}$ is a local minimum and $U^{1}$ the associated saddle point. Since all the local minima are considered, this gives in particular a canonical one to one correspondence between the small eigenvalues of $\Delta_{f, h}^{(0)}$ and the local minima of $f$.

According to the result of Witten which ensures that the number of small eigenvalues of $\Delta_{f, h}^{(p)}$ is $m_{p}$, the same kind of correspondence between these eigenvalues and the critical points of $f$ with index $p$ is expected for any $p$. As for the 0-forms, this correspondence means an association between the critical points with index $p$ and attached relevant critical points (probably with index $p+1$ or $p-1$ as it will be seen further).

The aim of this short paper is to notice that in the case of an oriented compact surface $\Omega$ without boundary, it is possible to obtain all the small eigenvalues of the self adjoint realization of $\Delta_{f, h}^{(p)}$, for $p \in\{0,1,2\}$. Moreover, as it is the case for the 0 -forms, there is actually a canonical association between the small eigenvalues of $\Delta_{f, h}^{(p)}$ and the critical points of $f$ with index $p$.

Before stating the main result, let us recall that $\Delta_{f, h}^{(p)}$ and $\Delta_{-f, h}^{(n-p)}$ are es- 
sentially dual operators under the action of the Hodge operator (see Proposition 4.1 further). The knowledge of the small eigenvalues of $\Delta_{f, h}^{(0)}$ then gives the small eigenvalues of $\Delta_{-f, h}^{(n)}$. The complete treatment of the case of a manifold with dimension 1 is therefore obvious.

The analysis done in $[\mathrm{HeKlNi}]$ requires an assumption which ensures that the exponentially small eigenvalues of $\Delta_{f, h}^{(0)}$ are simple, with different logarithmic equivalents as $h$ tends to $0^{1}$. As in [Nie], we are going to work here with a generic assumption which is slightly stronger in order to avoid some technical and unnecessary considerations.

Let us denote by $\mathcal{U}^{(p)}$, for $p \in\{0, \ldots, n\}$, the set of the critical points of $f$ with index $p$. Our generic assumption is:

Assumption 1.1. The function $f \in \mathcal{C}^{\infty}(\Omega, \mathbb{R})$ is a Morse function whose critical values are distinct. Moreover, the quantities $f(U)-f(V)$, where $U$ and $V$ are two different critical points of $f$, are also distinct.

Following this assumption, a one to one mapping $j_{01}$ (resp. $j_{21}$ ) can be defined from $\mathcal{U}^{(0)} \backslash\left\{U_{1}^{(0)}\right\}$ (resp. $\left.\mathcal{U}^{(2)} \backslash\left\{U_{1}^{(2)}\right\}\right)$, where $U_{1}^{(0)}$ is the global minimum (resp. maximum) of $f$, into the $\operatorname{set} \mathcal{U}^{(1)}$.

The minima (resp. maxima) are denoted by $U_{k_{0}}^{(0)}\left(\right.$ resp. $\left.U_{k_{2}}^{(2)}\right), k_{0} \in\left\{1, \ldots, m_{0}\right\}$ (resp. $k_{2} \in\left\{1, \ldots, m_{2}\right\}$ ), and the saddle points by $U_{j}^{(1)}, j \in\left\{1, \ldots, m_{1}\right\}$. The ordering of these extrema as well as the construction of the one to one mappings $j_{01}$ and $j_{21}$ will be specified further, in Section 3 .

Moreover, according to the following proposition, which is proved at the beginning of Section 4 ,

Proposition 1.2. The ranges of $j_{01}: \mathcal{U}^{(0)} \backslash\left\{U_{1}^{(0)}\right\} \rightarrow \mathcal{U}^{(1)}$ and $j_{21}: \mathcal{U}^{(2)} \backslash\left\{U_{1}^{(2)}\right\} \rightarrow \mathcal{U}^{(1)}$ are disjoint.

a one to one mapping $k$ from $\mathcal{U}^{(1)} \backslash\left\{U_{j}^{(1)}\right.$ s.t. $\left.U_{j}^{(1)} \notin \operatorname{Ran}\left(j_{01}\right), U_{j}^{(1)} \notin \operatorname{Ran}\left(j_{21}\right)\right\}$ into $\mathcal{U}^{(0)} \cup \mathcal{U}^{(2)}$ can be defined in the same spirit as $j_{01}$ and $j_{21}$. The labelling of the saddle points as well as the construction of $k$ are also specified in Section 3 .

Our main theorem, involving the definition of the previous injective maps, can now be stated:

\footnotetext{
${ }^{1}$ More precisely, such that the quantities $h \ln \lambda(h)$ and $h \ln \lambda^{\prime}(h)$ have different limits as $h \rightarrow 0$, where $\lambda(h)$ and $\lambda^{\prime}(h)$ are two distinct small eigenvalues of $\Delta_{f, h}^{(0)}$.
} 
Theorem 1.3. Under Assumption 1.1, there exists $h_{0}$ such that, for $h \in$ $\left(0, h_{0}\right]$ and $p \in\{0,1,2\}$, the spectrum in $\left[0, h^{\frac{3}{2}}\right)$ of $\Delta_{f, h}^{(p)}$ consists of $m_{p}$ eigenvalues,

$$
0=\lambda_{1}^{(p)}(h)=\cdots=\lambda_{b_{p}}^{(p)}(h)<\lambda_{b_{p}+1}^{(p)}(h)<\ldots<\lambda_{m_{p}}^{(p)}(h) .
$$

The above $m_{p}-b_{p}$ non zero eigenvalues are moreover simple, exponentially small, and admit a complete asymptotic expansion.

For $p \in\{0,2\}$, the Betti number $b_{p}$ is 1 , and, for every $k_{p}$ in $\left\{2, \ldots, m_{p}\right\}$,

$$
\lambda_{k_{p}}^{(p)}(h)=\frac{h}{\pi}\left|\widehat{\lambda}_{1}^{(p)}\right| \frac{\left|\operatorname{det} \operatorname{Hess} f\left(U_{k_{p}}^{(p)}\right)\right|^{\frac{1}{2}}}{\mid \operatorname{det} \operatorname{Hess} f\left(\left.U_{j_{p 1}\left(k_{p}\right)}^{(1)}\right|^{\frac{1}{2}}\right.} e^{-2 \frac{\left|f\left(U_{j_{p 1}\left(k_{p}\right)}^{(1)}\right)-f\left(U_{k_{p}}^{(p)}\right)\right|}{h}}\left(1+h c_{k_{p}}^{(p)}(h)\right),
$$

where $c_{k_{p}}^{(p)}(h) \sim \sum_{m=0}^{\infty} h^{m} c_{k_{p}, m}^{(p)}$ and $\hat{\lambda}_{1}^{(0)}$ (resp. $\hat{\lambda}_{1}^{(2)}$ ) is the negative (resp. positive) eigenvalue of $\operatorname{Hess} f\left(U_{j_{01}\left(k_{0}\right)}^{(1)}\right)$ (resp. Hess $f\left(U_{j_{21}\left(k_{2}\right)}^{(1)}\right)$ ).

For $p=1$, the eigenvalue 0 has multiplicity $b_{1}$ and for every $j$ in $\left\{b_{1}+\right.$ $\left.1, \ldots, m_{1}\right\}$,

$$
\lambda_{j}^{(1)}(h)=\frac{h}{\pi}\left|\widehat{\lambda}_{1}\right| \frac{\left|\operatorname{det} \operatorname{Hess} f\left(U_{k(j)}^{(p)}\right)\right|^{\frac{1}{2}}}{\left|\operatorname{det} \operatorname{Hess} f\left(U_{j}^{(1)}\right)\right|^{\frac{1}{2}}} e^{-2 \frac{\left|f\left(U_{k(j)}^{(p)}\right)-f\left(U_{j}^{(1)}\right)\right|}{h}}\left(1+h c_{j}^{(1)}(h)\right),
$$

where $p \in\{0,2\}, c_{j}^{(1)}(h) \sim \sum_{m=0}^{\infty} h^{m} c_{j, m}^{(1)}$, and, if $k(j) \in \mathcal{U}^{(0)} \quad \operatorname{resp} . k(j) \in$ $\left.\mathcal{U}^{(2)}\right), \widehat{\lambda}_{1}$ is the negative (resp. positive) eigenvalue of Hess $f\left(U_{j}^{(1)}\right)$.

Let us make a few comments about this theorem. First, the result concerning the small eigenvalues of $\Delta_{f, h}^{(0)}$ is nothing but the main result of [HeKlNi]. The simplicity of the non zero eigenvalues of $\Delta_{f, h}^{(0)}$ and $\Delta_{f, h}^{(2)}$ is moreover obvious, according to the theorem and to Assumption 1.1 (look indeed at the quantities involved in the exponential term). The proof of the result for $\Delta_{f, h}^{(2)}$ only lies on a simple trick involving the Hodge operator: the small eigenvalues of $\Delta_{f, h}^{(2)}$ are the small eigenvalues of $\Delta_{-f, h}^{(0)}$.

Using some elementary results of linear algebra, it is then quite easy to show that the set of the $m_{1}-b_{1}$ non zero eigenvalues of $\Delta_{f, h}^{(1)}$ is

$$
\left\{\lambda_{k_{0}}^{(0)}(h), \lambda_{k_{2}}^{(2)}(h) \text {, with }\left(k_{0}, k_{2}\right) \in\left\{2, \ldots, m_{0}\right\} \times\left\{2, \ldots, m_{2}\right\}\right\} .
$$

The proper writing of its non zero eigenvalues is therefore a simple consequence of the definition of the one to one mapping $k$. Owing to the writing of 
$\lambda_{k_{p}}^{(p)}(h)$ in the theorem, it suffices indeed to define $\lambda_{j_{p 1}\left(k_{p}\right)}^{(1)}$ as $\lambda_{k_{p}}^{(p)}$, for $p \in\{0,2\}$ and $k_{p} \in\left\{2, \ldots, m_{p}\right\}$. In other words, for $j>b_{1}, \lambda_{j}^{(1)}=\lambda_{k_{p}}^{(p)}$, where $k_{p}=k(j)$.

The proof of Theorem 1.3 relies on the main result of [HeKlNi], Hodge theory and some primary results of linear algebra and homology theory. In particular, the finest part of the proof is the one to one correspondence between the saddle points of $f$ and the small eigenvalues of $\Delta_{f, h}^{(1)}$ (i.e. the proof of Proposition 1.2).

This result will be proved in the fourth section. The second one is devoted to the recalling of some generalities about Witten Laplacians and the third one to the ordering of the critical points as well as to the construction of the one to one mappings $j_{01}, j_{21}$ and $k$.

Remark 1.4. According to the above comments, we do not need Proposition 1.2 and the introduction of the one to one mapping $k$ if we only want to show that all the small eigenvalues of $\Delta_{f, h}^{(1)}$ are obtained.

Nevertheless, without Proposition 1.2, it is not possible to ensure that the canonical pairing between these small eigenvalues and the saddle points (with index 1) is a one to one correspondence. By canonical pairing, we mean the pair $\left(\lambda_{j}^{(1)}, U_{j}^{(1)}\right)$, where $U_{j}^{(1)}$ is the critical point involved in the writing of $\lambda_{j}^{(1)}$ (in the exponential term). It would indeed be a priori possible that $\lambda_{j}^{(1)}>0$ and $\lambda_{j^{\prime}}^{(1)}>0, j \neq j^{\prime}$, involve in their writing the same saddle point $U_{j}^{(1)}$.

\section{Generalities about Witten Laplacians}

Let $\left(\Omega, g_{0}\right)$ be a $\mathcal{C}^{\infty}$ connected compact oriented Riemannian manifold without boundary and with dimension $n \in \mathbb{N}^{*}, g_{0}$ denoting the given Riemannian metric on $\Omega$.

The cotangent (resp. tangent) bundle on $\Omega$ is denoted by $T^{*} \Omega$ (resp. $T \Omega$ ) and the exterior fiber bundle by $\Lambda T^{*} \Omega=\oplus_{p=0}^{n} \Lambda^{p} T^{*} \Omega\left(\right.$ resp. $\left.\Lambda T \Omega=\oplus_{p=0}^{n} \Lambda^{p} T \Omega\right)$. The space of the $\mathcal{C}^{\infty}, L^{2}, \mathcal{H}^{s}$ sections in $\Lambda T^{*} \Omega$ are respectively denoted by $\mathcal{C}^{\infty}\left(\Omega ; \Lambda T^{*} \Omega\right), L^{2}\left(\Omega ; \Lambda T^{*} \Omega\right)$, and $\mathcal{H}^{s}\left(\Omega ; \Lambda T^{*} \Omega\right)$. Let us also recall that the $L^{2}$ spaces are those associated with the unit volume form for the Riemannian structure on $\Omega$.

Let $d$ be the exterior differential on $\mathcal{C}^{\infty}\left(\Omega ; \Lambda T^{*} \Omega\right)$,

$$
d^{(p)}: \mathcal{C}^{\infty}\left(\Omega ; \Lambda^{p} T^{*} \Omega\right) \rightarrow \mathcal{C}^{\infty}\left(\Omega ; \Lambda^{p+1} T^{*} \Omega\right)
$$


and $d^{*}$ its formal adjoint with respect to the $L^{2}$-scalar product inherited from the Riemannian structure,

$$
d^{(p), *}: \mathcal{C}^{\infty}\left(\Omega ; \Lambda^{p+1} T^{*} \Omega\right) \rightarrow \mathcal{C}^{\infty}\left(\Omega ; \Lambda^{p} T^{*} \Omega\right)
$$

Set, for a function $f \in \mathcal{C}^{\infty}(\Omega ; \mathbb{R})$ and $h>0$, the distorted operators defined on $\mathcal{C}^{\infty}\left(\Omega ; \Lambda T^{*} \Omega\right)$ :

$$
d_{f, h}=e^{-f(x) / h}(h d) e^{f(x) / h} \quad \text { and } \quad d_{f, h}^{*}=e^{f(x) / h}\left(h d^{*}\right) e^{-f(x) / h} .
$$

The Witten Laplacian is the differential operator defined on $\mathcal{C}^{\infty}\left(\Omega ; \Lambda T^{*} \Omega\right)$ by:

$$
\Delta_{f, h}=d_{f, h}^{*} d_{f, h}+d_{f, h} d_{f, h}^{*}=\left(d_{f, h}+d_{f, h}^{*}\right)^{2} .
$$

The last equality follows from the property $d d=d^{*} d^{*}=0$ which implies:

$$
d_{f, h} d_{f, h}=d_{f, h}^{*} d_{f, h}^{*}=0 .
$$

It means, by restriction to the $p$-forms in $\mathcal{C}^{\infty}\left(\Omega ; \Lambda^{p} T^{*} \Omega\right)$ :

$$
\Delta_{f, h}^{(p)}=d_{f, h}^{(p), *} d_{f, h}^{(p)}+d_{f, h}^{(p-1)} d_{f, h}^{(p-1), *} .
$$

The Hodge operator $\star$,

$$
\star: \mathcal{C}^{\infty}\left(\Omega ; \Lambda^{p} T^{*} \Omega\right) \rightarrow \mathcal{C}^{\infty}\left(\Omega ; \Lambda^{n-p} T^{*} \Omega\right),
$$

is locally defined in a pointwise orthonormal frame $\left(E_{1}, \ldots, E_{n}\right)$ by:

$$
\left(\star \omega_{x}\right)\left(E_{\sigma(p+1)}, \ldots, E_{\sigma(n)}\right)=\varepsilon(\sigma) \omega_{x}\left(E_{\sigma(1)}, \ldots, E_{\sigma(p)}\right),
$$

for $\omega_{x} \in \Lambda^{p} T_{x}^{*} \Omega$ and with any permutation $\sigma \in \Sigma(n)$ of $\{1, \ldots, n\}$ preserving $\{1, \ldots, p\}(\varepsilon(\sigma)$ denotes the signature of $\sigma)$.

This operator is essentially mentionned here in order to recall the following formulas wich will be useful further:

$$
\begin{gathered}
\star\left(\star \omega_{x}\right)=(-1)^{p(n-p)} \omega_{x}, \quad \forall \omega_{x} \in \Lambda^{p} T_{x}^{*} \Omega, \\
\left\langle\omega_{1} \mid \omega_{2}\right\rangle_{\Lambda^{p} L^{2}}=\int_{\Omega} \omega_{1} \wedge \star \overline{\omega_{2}}, \quad \forall \omega_{1}, \omega_{2} \in \Lambda^{p} L^{2}, \\
\star d^{*,(p-1)}=(-1)^{p} d^{(n-p)} \star, \quad \star d^{(p)}=(-1)^{p+1} d^{*,(n-p-1)} \star .
\end{gathered}
$$

Let us now recall a first result about the localization of the small eigenvalues of $\Delta_{f, h}^{(p)}$ used by E. Witten in his proof of the Morse inequalities (c.f. [Wit][HeSj4]). 
Theorem 2.1. For $p \in\{0, \ldots, n\}$, the Witten Laplacian $\Delta_{f, h}^{(p)}$ starting from $\mathcal{C}^{\infty}\left(\Omega ; \Lambda^{p} T^{*} \Omega\right)$ is essentially self adjoint on $L^{2}\left(\Omega ; \Lambda^{p} T^{*} \Omega\right)$ and its self adjoint realization, still denoted by $\Delta_{f, h}^{(p)}$, has for domain the Sobolev space $\mathcal{H}^{2}\left(\Omega ; \Lambda^{p} T^{*} \Omega\right)$.

Moreover, if $f$ is a Morse function, there exists $h_{0}>0$, such that, for $h \in\left(0, h_{0}\right], \Delta_{f, h}^{(p)}$ has the following property: for $p \in\{0, \ldots, n\}$, the spectral subspace $\operatorname{Ran} 1_{\left[0, h^{3 / 2}\right)}\left(\Delta_{f, h}^{(p)}\right)$ has rank $m_{p}(f)$, the number of critical points of $f$ with index $p$. The kernel Ker $\Delta_{f, h}^{(p)}$ has moreover rank $b_{p}$, the p-th Betti number of the manifold $\Omega$.

Let us also recall the Witten complex structure, which is the core of Witten's approach to Morse inequalities.

Denote by $F^{(p)}$ the spectral subspace Ran $1_{\left[0, h^{3 / 2}\right)}\left(\Delta_{f, h}^{(p)}\right)$. Then, $B_{f, h}^{(p)}=\left.d_{f, h}^{(p)}\right|_{F^{(p)}}$ and $B_{f, h}^{(p), *}=\left.d_{f, h}^{(p-1), *}\right|_{F^{(p)}}$ define two complexes of finite dimensional spaces:

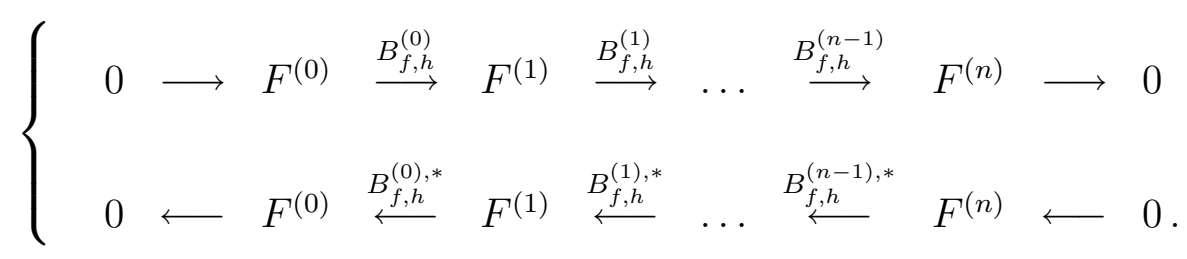

Furthermore, this complex structure linked to Theorem 2.1 directly leads to the Morse inequalities:

$$
\left\{\begin{array}{l}
\sum_{p=0}^{k}(-1)^{k-p} m_{p}(f) \geq \sum_{p=0}^{k}(-1)^{k-p} b_{p}, 0 \leq k<n \\
\sum_{p=0}^{n}(-1)^{p} m_{p}(f)=\sum_{p=0}^{n}(-1)^{p} b_{p} .
\end{array}\right.
$$

\section{Labelling of the critical points and defini- tion of the injective maps $j_{01}, j_{21}$ and $k$}

\subsection{Labelling of the extrema, definition of $j_{01}$ and $j_{21}$}

The ordering of the minima and the construction/definition of the one to one mapping $j_{01}$ are done as follows (see also [Nie] or [HeKlNi] for a slightly weaker assumption than our generic one). Let us also recall that they correspond, in the probability framework, to the ordering of exit times, according to $[\mathrm{BoEcGaKl}][\mathrm{BoGaKl}]$.

Ordering of the minima and definition of $j_{01}$ 
Remember first that for a real number $\lambda$, the number of connected components of the sublevel set $\{f<\lambda\}$ is the dimension of its 0 -th order homology group $b_{0}(\{f<\lambda\})=\operatorname{dim} H_{0}(\{f<\lambda\})$.

1) Set $U_{1}^{(0)}=\min _{x \in \Omega} f, z_{1}=\infty, f\left(z_{1}\right)=+\infty$ and consider $b_{0}(\{f<\lambda\})$, for $\lambda$ decreasing from $f\left(z_{1}\right)=+\infty$.

2) When $U_{k_{0}}^{(0)}$ and $z_{k_{0}}$ are defined for $k_{0}=1, \ldots, K_{0}-1$, decrease $\lambda$ from $f\left(z_{K_{0}-1}\right)$ until $b_{0}(\{f<\lambda\})$ increases by 1 . Denote by $\lambda_{K_{0}}$ this value.

3) Due to the structure of the level sets of a Morse function, there exists a point in $\mathcal{U}^{(1)}$, which has to be unique according to Assumption 1.1, that we denote by $z_{K_{0}}$, satisfying $f\left(z_{K_{0}}\right)=\lambda_{K_{0}}$. Denote then by $U_{K_{0}}^{(0)}$ the global minimum of the new connected component.

4) Iterate 2) and 3) until all the local minima have been considered.

5) Lastly, permute the $k_{0}$ 's to make the sequence $\left(f\left(z_{k_{0}}\right)-f\left(U_{k_{0}}^{(0)}\right)\right)_{k_{0} \in\left\{1, \ldots, m_{0}\right\}}$ strictly decreasing, which is possible by Assumption 1.1.

6) The one to one mapping $j_{01}$ is defined from $\mathcal{U}^{(0)} \backslash\left\{U_{1}^{(0)}\right\}$ into $\mathcal{U}^{(1)}$ by:

$$
\forall k_{0} \geq 2, U_{j_{01}\left(k_{0}\right)}^{(1)}=z_{k_{0}} .
$$

In order to compute the small eigenvalues of $\Delta_{f, h}^{(2)}$, we are going to apply the results of [HeKlNi] to the Witten Laplacian $\Delta_{-f, h}^{(0)}$, whose minima and saddle points (with index 1) are nothing but the maxima and saddle points (with index 1) of $f$.

The construction of $j_{21}$ is thereby the same as the one of $j_{01}$, after having replaced $f$ by $-f$. An equivalent way to see it is to work with $f$ again, but to consider the connected components of the upperlevel sets of $f$ instead of its sublevel sets and to make $\lambda$ increase:

\section{Ordering of the maxima and definition of $j_{21}$}

1) Set $U_{1}^{(2)}=\max _{x \in \Omega} f, z_{1}=\infty, f\left(z_{1}\right)=-\infty$ and consider $b_{0}(\{f>\lambda\})$, for $\lambda$ increasing from $f\left(z_{1}\right)=-\infty$.

2) When $U_{k_{2}}^{(2)}$ and $z_{k_{2}}$ are defined for $k_{2}=1, \ldots, K_{2}-1$, increase $\lambda$ from $f\left(z_{K_{2}-1}\right)$ until $b_{0}(\{f>\lambda\})$ increases by 1 . Denote by $\lambda_{K_{2}}$ this value. 
3) By Assumptions 1.1, there exists a unique point in $\mathcal{U}^{(1)}$, that we denote by $z_{K_{2}}$, satisfying $f\left(z_{K_{2}}\right)=\lambda_{K_{2}}$. Denote then by $U_{K_{2}}^{(2)}$ the global maximum of the new connected component.

4) Iterate 2) and 3) until all the local maxima have been considered.

5) Lastly, permute the $k_{2}$ 's to make the sequence $\left(f\left(U_{k_{2}}^{(2)}-f\left(z_{k_{2}}\right)\right)\right)_{k_{2} \in\left\{1, \ldots, m_{2}\right\}}$ strictly decreasing, which is possible by Assumption 1.1.

6) The one to one mapping $j_{21}$ is defined from $\mathcal{U}^{(2)} \backslash\left\{U_{1}^{(2)}\right\}$ into $\mathcal{U}^{(1)}$ by:

$$
\forall k_{2} \geq 2, U_{j_{21}\left(k_{2}\right)}^{(1)}=z_{k_{2}}
$$

\subsection{Labelling of the saddle points and definition of $k$}

Let us now label the saddle points $U_{j}^{(1)}, j \in\left\{1, \ldots, m_{1}\right\}$, in order to define the one to one mapping $k$ from $\mathcal{U}^{(1)}$ into $\mathcal{U}^{(0)} \cup \mathcal{U}^{(2)}$.

Owing to Proposition 1.2 which ensures that the ranges of $j_{01}$ and $j_{02}$ are disjoint, the subset

$$
\left\{U_{j_{p 1}\left(k_{p}\right)}^{(1)}, p \in\{0,2\}, k_{p} \in\left\{2, \ldots, m_{p}\right\}\right\} \text { of } \mathcal{U}^{(1)}
$$

consists of $\left(m_{0}-1\right)+\left(m_{2}-1\right)$ saddle points, or equivalently, due to the Morse inequalities, of $m_{1}-b_{1}$ saddle points.

The $b_{1}$ points of $\mathcal{U}^{(1)}$ which are not reached by both $j_{01}$ and $j_{21}$ are denoted by $U_{1}^{(1)}, \ldots, U_{b_{1}}^{(1)}$. The ordering of the remainding saddle points and the construction of the one to one mapping $k$ are done as follows.

Once $U_{j}^{(1)}$ and $k(j)$ are defined for $j=b_{1}+1, \ldots, J-1, U_{J}^{(1)}$ and $k(J)$ are chosen in the following way:

- take $J=j_{p 1}\left(K_{p}\right)$ such as $\left|f\left(U_{K_{p}}^{(p)}\right)-f\left(U_{j_{p 1}\left(K_{p}\right)}^{(1)}\right)\right|$ is the unique minimizer of the set $\left\{\left|f\left(U_{k_{p}}^{(p)}\right)-f\left(U_{j_{p 1}\left(k_{p}\right)}^{(1)}\right)\right|\right\}$, where $p \in\{0,2\}, 2 \leq k_{p} \leq m_{p}$, and $\left(k_{0}, k_{2}\right) \notin\left\{k\left(b_{1}+1\right), \ldots, k(J-1)\right\}$,

- set then $k(J)=K_{p}$.

The one to one mapping $k$ is therefore defined from $\mathcal{U}^{(1)} \backslash\left\{U_{1}^{(1)}, \ldots, U_{b_{1}}^{(1)}\right\}$ into $\mathcal{U}^{(0)} \cup \mathcal{U}^{(2)}$. It is even a bijection from $\mathcal{U}^{(1)} \backslash\left\{U_{1}^{(1)}, \ldots, U_{b_{1}}^{(1)}\right\}$ onto $\left(\mathcal{U}^{(0)} \backslash\left\{U_{1}^{(0)}\right\}\right) \cup\left(\mathcal{U}^{(2)} \backslash\left\{U_{1}^{(2)}\right\}\right)$. 
Remark 3.1. Actually, the ordering of the saddle points $U_{j}^{(1)}$ 's $\left(j>b_{1}\right)$ and the construction of the one to one mapping $k$ are just made in such way as the two following conditions are fulfilled:

- $k$ is the inverse of $j_{p 1}$ on the range of $j_{p 1}$, for $p \in\{0,2\}$,

- the sequence $\left(\left|f\left(U_{k(j)}^{(p)}\right)-f\left(U_{j}^{(1)}\right)\right|\right)_{j>b_{1}}$ is strictly decreasing.

\section{Proofs and remarks}

Proof of Proposition 1.2.

Let us first define, for $a \in \mathbb{R}$,

$$
\Omega_{-}^{a}:=\{x \in \Omega, f(x) \leq a\} \quad \text { and } \quad \Omega_{+}^{a}:=\{x \in \Omega, f(x) \geq a\} .
$$

If $a$ is not a critical value of $f$, the sets $\Omega_{-}^{a}$ and $\Omega_{+}^{a}$ are two oriented compact 2-manifolds with boundary $\Omega^{a}:=\{x \in \Omega, f(x)=a\}$, which is an oriented compact 1-manifold without boundary. To be more precise, $\Omega^{a}$ is homotopic to a union of disjoint circles. The number of connected components of these sets are respectively denoted by $b_{0}\left(\Omega_{-}^{a}\right), b_{0}\left(\Omega_{+}^{a}\right)$ and $b_{0}\left(\Omega^{a}\right)$. According to the usual Morse theory (see e.g. [Mil] or [Gra]), $b_{0}\left(\Omega_{\mp}^{a}\right)$ is constant when $a$ stays between two critical values of $f$.

Take now $U^{1}$ a critical point of $f$ with index 1 and $a<b$ two real numbers such that $U^{1}$ is the only critical point of $f$ in $f^{-1}([a, b])$. This is possible by Assumption 1.1 which ensures that the critical values of $f$ are distinct.

According again to the usual Morse theory (see again [Mil] or [Gra]), if we denote by $b_{1}\left(\Omega_{-}^{a}\right)$ (resp. $b_{1}\left(\Omega_{+}^{a}\right)$ ) the dimension of the first order homology group $H_{1}\left(\Omega_{-}^{a}\right)$ (resp. $\left.H_{1}\left(\Omega_{+}^{a}\right)\right)$, two possibilities can happen:

$$
\begin{gathered}
\left\{\begin{array} { l } 
{ b _ { 0 } ( \Omega _ { - } ^ { a } ) = b _ { 0 } ( \Omega _ { - } ^ { b } ) + 1 } \\
{ b _ { 1 } ( \Omega _ { - } ^ { a } ) = b _ { 1 } ( \Omega _ { - } ^ { b } ) }
\end{array} \text { or } \left\{\begin{array}{l}
b_{0}\left(\Omega_{-}^{a}\right)=b_{0}\left(\Omega_{-}^{b}\right) \\
b_{1}\left(\Omega_{-}^{a}\right)=b_{1}\left(\Omega_{-}^{b}\right)-1
\end{array}\right.\right. \\
\left(\operatorname { r e s p } \left\{\begin{array}{l}
b_{0}\left(\Omega_{+}^{a}\right)+1=b_{0}\left(\Omega_{+}^{b}\right) \\
b_{1}\left(\Omega_{+}^{a}\right)=b_{1}\left(\Omega_{+}^{b}\right)
\end{array} \text { or }\left\{\begin{array}{l}
b_{0}\left(\Omega_{+}^{a}\right)=b_{0}\left(\Omega_{+}^{b}\right) \\
b_{1}\left(\Omega_{+}^{a}\right)-1=b_{1}\left(\Omega_{+}^{b}\right)
\end{array}\right) .\right.\right.
\end{gathered}
$$

Therefore, four cases are a priori possible and owing to the definitions of the one to one mappings $j_{01}$ and $j_{21}$, Proposition 1.2 amounts to show that one of these a priori possible cases cannot happen, or equivalently that the two following propositions cannot occur simultaneously (but they can a priori both not happen):

$$
b_{0}\left(\Omega_{-}^{a}\right)=b_{0}\left(\Omega_{-}^{b}\right)+1,
$$




$$
b_{0}\left(\Omega_{+}^{b}\right)=b_{0}\left(\Omega_{+}^{a}\right)+1 .
$$

Let us also notice that this incompatibility is implied by the two following implications:

$$
\begin{array}{ll}
\text { If } \quad b_{0}\left(\Omega_{-}^{a}\right)=b_{0}\left(\Omega_{-}^{b}\right)+1, \quad \text { then } \quad b_{0}\left(\Omega^{a}\right)>b_{0}\left(\Omega^{b}\right), \\
\text { If } \quad b_{0}\left(\Omega_{+}^{b}\right)=b_{0}\left(\Omega_{+}^{a}\right)+1, \quad \text { then } \quad b_{0}\left(\Omega^{b}\right)>b_{0}\left(\Omega^{a}\right) .
\end{array}
$$

These implications are obviously "symmetric" and we are only going to prove (4.3).

Let us then assume that Equation (4.1) is satisfied and let us denote by $\Omega_{-}^{b, 1}, \ldots, \Omega_{-}^{b, N}\left(\operatorname{resp} . \quad \Omega_{-}^{a, 1}, \ldots, \Omega_{-}^{a, N+1}\right)$ the $N:=b_{0}\left(\Omega_{-}^{b}\right)(\operatorname{resp} . \quad N+1=$ $\left.b_{0}\left(\Omega_{-}^{a}\right)\right)$ connected components of $\Omega_{-}^{b}$ (resp. $\Omega_{-}^{a}$ ), with $U^{(1)} \in \Omega_{-}^{b, N}$.

Since $U^{1}$ is the only critical point of $f$ in $f^{-1}([a, b])$, according to Theorem 3.1 of [Mil] p. 12, these connected components can be ordered such as, for $k$ in $\{1, \ldots, N-1\}, \Omega_{-}^{a, k}$ is a deformation retract of $\Omega_{-}^{b, k}$ (nothing happens for these components since they do not contain any critical point in $\left.f^{-1}([a, b])\right)$. In particular, the following relation is satisfied,

$$
\forall k \in\{1, \ldots, N-1\}, \quad b_{0}\left(\Omega^{b, k}\right)=b_{0}\left(\Omega^{a, k}\right),
$$

and the proof amounts to check that

$$
b_{0}\left(\Omega^{a, N}\right)+b_{0}\left(\Omega^{a, N+1}\right)>b_{0}\left(\Omega^{b, N}\right) .
$$

According to [Gra] pp. 60-61, one of the three following equalities occurs,

$$
b_{0}\left(\Omega^{a, N}\right)+b_{0}\left(\Omega^{a, N+1}\right)=b_{0}\left(\Omega^{b, N}\right) \pm 1 \text { or } b_{0}\left(\Omega^{a, N}\right)+b_{0}\left(\Omega^{a, N+1}\right)=b_{0}\left(\Omega^{b, N}\right),
$$

and the fact that $\Omega$ is oriented ensures that the third case cannot happen (see Proposition 5 of [Gra], p. 81).

It appears now clearly that, if the number of connected components of the boundary $\{f=\lambda\}$ decreases when the critical value $f\left(U^{1}\right)$ is crossed (for $\lambda=b$ then $\lambda=a$ ), i.e. if

$$
b_{0}\left(\Omega^{a, N}\right)+b_{0}\left(\Omega^{a, N+1}\right)=b_{0}\left(\Omega^{b, N}\right)-1,
$$

then the number of connected components of $\{f \leq \lambda\}$ cannot increase, i.e. $\Omega^{b, N}$ does not give two connected components, $\Omega^{a, N}$ and $\Omega^{a, N+1}$. Hence the only possibility is $b_{0}\left(\Omega^{a, N}\right)+b_{0}\left(\Omega^{a, N+1}\right)=b_{0}\left(\Omega^{b, N}\right)+1$, which implies (4.5), and Proposition 1.2 is proven. 
The rest of the proof of Theorem 1.3 is quite easy if we keep in mind the Morse inequalities and the following results.

Lemma 4.1. (Stated for a compact manifold with dimension $n \in \mathbb{N}^{*}$ )

Let $u_{p}$ be an eigenvector of $\Delta_{f, h}^{(p)}$ associated with the eigenvalue $\lambda_{p}$, for some $p \in\{0, \ldots, n\}$. Then $\star u_{p}$ is an eigenvector of $\Delta_{-f, h}^{(n-p)}$ associated with the eigenvalue $\lambda_{p}$. The multiplicity of $\lambda_{p}$ is moreover the same for $\Delta_{f, h}^{(p)}$ and $\Delta_{-f, h}^{(n-p)}$ since the Hodge operator $\star$ is bijective.

Proof. Coming back to the definitions of $d_{f, h}$ and $d_{f, h}^{*}$ (see (2.1)), and owing to Equation (2.7), let us write the following relations:

$$
\star d_{f, h}^{*,(p-1)}=(-1)^{p} d_{-f, h}^{(n-p)} \star \text { and } \star d_{f, h}^{(p)}=(-1)^{p+1} d_{-f, h}^{*,(n-p-1)} \star .
$$

This implies the following formal relations which are the main point of the proof:

$$
\star \Delta_{f, h}^{(p)}=\Delta_{-f, h}^{(n-p)} \star \quad \text { and } \quad \star \Delta_{f, h}^{(n-p)}=\Delta_{-f, h}^{(p)} \star .
$$

Hence the proof only comes down to check the action of the Hodge operator $\star$ on the operator domain, $\mathcal{H}^{2}\left(\Omega ; \Lambda T^{*} \Omega\right)$, which is actually preserved under its action.

Lemma 4.2. (Stated for a compact manifold with dimension $n \in \mathbb{N}^{*}$ ) Let $u_{p}$ be an eigenvector of $\Delta_{f, h}^{(p)}$ associated with the eigenvalue $\lambda_{p}$, for some $p \in\{0, \ldots, n\}$. Then, if $\lambda_{p} \neq 0$, one of the two following cases occurs:

- either $d_{f, h} u_{p}$ is an eigenvector of $\Delta_{f, h}^{(p+1)}$ associated with the eigenvalue $\lambda_{p}$,

- or $d_{f, h}^{*} u_{p}$ is an eigenvector of $\Delta_{f, h}^{(p-1)}$ associated with the eigenvalue $\lambda_{p}$.

Moreover, a basis of $\operatorname{Ker}\left(\Delta_{f, h}^{(p)}-\lambda_{p}\right)$ can be chosen such as, for each element of the basis, only one of the above cases occurs, the other vector being 0 .

Proof. Let us first recall, due to the ellipticity of the Witten Laplacian, that an eigenvector $u_{p}$ of $\Delta_{f, h}^{(p)}$ is in $\mathcal{C}^{\infty}\left(\Omega ; \Lambda T^{*} \Omega\right)$. Hence, there is no problem of regularity. Assume now that both $d_{f, h} u_{p}$ and $d_{f, h}^{*} u_{p}$ are zero and let us show that it leads to a contradiction.

From the relation

$$
\Delta_{f, h}^{(p)} u_{p}=\lambda_{p} u_{p}
$$


we obtain, looking at the scalar product with $u_{p}$ :

$$
0=\left\|d_{f, h} u_{p}\right\|^{2}+\left\|d_{f, h}^{*} u_{p}\right\|^{2}=\lambda_{p}\left\|u_{p}\right\|^{2} .
$$

This is a contradiction since $u_{p} \neq 0$ and $\lambda_{p} \neq 0$.

The rest of the proof is a consequence of the complex structure and Hodge theory. Assume that $\lambda_{p}$ is an eigenvalue of $\Delta_{f, h}^{(p)}$ and take $\left(u_{p+1}^{1}, \ldots, u_{p+1}^{\ell}\right)$ (resp. $\left.\left(u_{p-1}^{1}, \ldots, u_{p-1}^{\ell^{\prime}}\right)\right)$ an orthogonal basis of $\operatorname{Ran} d_{f, h}^{(p)}\left(\operatorname{Ker}\left(\Delta_{f, h}^{(p)}-\lambda_{p}\right)\right)$ $\left(\right.$ resp. $\left.\operatorname{Ran} d_{f, h}^{(p-1), *}\left(\operatorname{Ker}\left(\Delta_{f, h}^{(p)}-\lambda_{p}\right)\right)\right)$. The following relations are hence satisfied:

$$
\forall 1 \leq k \leq \ell, d_{f, h} u_{p+1}^{k}=0 \quad \text { and } \quad \forall 1 \leq k \leq \ell^{\prime}, d_{f, h}^{*} u_{p-1}^{k}=0 .
$$

Owing to the first part of the proposition, the set

$$
\left(d_{f, h}^{*} u_{p+1}^{1}, \ldots, d_{f, h}^{*} u_{p+1}^{\ell}, d_{f, h} u_{p-1}^{1}, \ldots, d_{f, h} u_{p-1}^{\ell^{\prime}}\right)
$$

then consists of $\ell+\ell^{\prime}$ non zero eigenvectors of $\Delta_{f, h}^{(p)}$ associated with $\lambda_{p}$. Since each of these vectors is obviously cancelled by $d_{f, h}$ or $d_{f, h}^{*}$, for proving the end of the proposition, it is sufficient to show that they form a basis of $\operatorname{Ker}\left(\Delta_{f, h}^{(p)}-\lambda_{p}\right)$.

This family of vectors is linearly independent since orthogonal:

$$
\begin{aligned}
\forall 1 \leq k \neq k^{\prime} \leq \ell, \forall 1 \leq j & \neq j^{\prime} \leq \ell^{\prime}, \\
\left\langle d_{f, h}^{*} u_{p+1}^{k}, d_{f, h}^{*} u_{p+1}^{k^{\prime}}\right\rangle & =\left\langle\Delta_{f, h}^{(p+1)} u_{p+1}^{k}, u_{p+1}^{k^{\prime}}\right\rangle=\lambda_{p}\left\langle u_{p+1}^{k}, u_{p+1}^{k^{\prime}}\right\rangle=0, \\
\left\langle d_{f, h} u_{p-1}^{j}, d_{f, h} u_{p-1}^{j^{\prime}}\right\rangle & =\left\langle\Delta_{f, h}^{(p-1)} u_{p-1}^{j}, u_{p-1}^{j^{\prime}}\right\rangle=\lambda_{p}\left\langle u_{p-1}^{j}, u_{p-1}^{j^{\prime}}\right\rangle=0, \\
\left\langle d_{f, h}^{*} u_{p+1}^{k}, d_{f, h} u_{p-1}^{j}\right\rangle & =\left\langle u_{p+1}^{k}, d_{f, h} d_{f, h} u_{p-1}^{j}\right\rangle=0 .
\end{aligned}
$$

To conclude, it reminds only to check that this family generates $\operatorname{Ker}\left(\Delta_{f, h}^{(p)}-\lambda_{p}\right)$. Take then $v_{p}$ in $\operatorname{Ker}\left(\Delta_{f, h}^{(p)}-\lambda_{p}\right)$ with $v_{p}$ orthogonal to $d_{f, h}^{*} u_{p+1}^{1}, \ldots, d_{f, h}^{*} u_{p+1}^{\ell}$ and to $d_{f, h} u_{p-1}^{1}, \ldots, d_{f, h} u_{p-1}^{\ell^{\prime}}$, and show that $v_{p}$ is zero. For all $k$ in $\{1, \ldots, \ell\}$, the relation

$$
0=\left\langle v_{p}, d_{f, h}^{*} u_{p+1}^{k^{\prime}}\right\rangle=\left\langle d_{f, h} v_{p}, u_{p+1}^{k^{\prime}}\right\rangle
$$

implies that $d_{f, h}^{(p)} v_{p}$ belongs to $\operatorname{Ran} d_{f, h}^{(p)}\left(\operatorname{Ker}\left(\Delta_{f, h}^{(p)}-\lambda_{p}\right)\right)^{\perp}$. Hence $d_{f, h}^{(p)} v_{p}$ is zero, since $d_{f, h}^{(p)} v_{p}$ obviously belongs to $\operatorname{Ran} d_{f, h}^{(p)}\left(\operatorname{Ker}\left(\Delta_{f, h}^{(p)}-\lambda_{p}\right)\right)$. By the same argument, $d_{f, h}^{(p-1), *} v_{p}$ is also zero. This implies the nullity of $v_{p}$ and ends up the proof of Lemma 4.2. 
Let us now go back to the case of a manifold with dimension 2. The last lemma directly implies the following result:

Lemma 4.3. Let $\Delta_{f, h}$ denotes the self adjoint realization of the Witten Laplacian. The set of the non zero eigenvalues of $\Delta_{f, h}^{(1)}$ (counted with multiplicity) is the union of the set of the non zero eigenvalues of $\Delta_{f, h}^{(0)}$ with the set of the non zero eigenvalues of $\Delta_{f, h}^{(2)}$.

Let us now prove Theorem 1.3.

Proof of Theorem 1.3. The result given for $\Delta_{f, h}^{(0)}$ is the main result of [HeKlNi] and the result given for $\Delta_{f, h}^{(2)}$ is a direct consequence of [HeKlNi] (with the Morse function $-f$ ) and Lemma 4.1. Recall moreover that the number of small eigenvalues of $\Delta_{-f, h}^{(0)}$ is actually $m_{2}$.

Owing to Lemma 4.3 , we obtain all the non zero eigenvalues of $\Delta_{f, h}^{(1)}$. The ordering of the saddle points as well as the definition of the one to one mapping $k$ then allow us to write the asymptotic formulas stated in Theorem 1.3 for $\Delta_{f, h}^{(1)}$.

Remark 4.4. 1) The trick used here unfortunately does not work for higher dimensions, not even for $n=3$. In this last case, we cannot, following only the previous analysis, determinate all the non zero smallest eigenvalues of $\Delta_{f, h}^{(1)}$ and $\Delta_{f, h}^{(2)}$, but we can nevertheless obtain $m_{0}-b_{0}$ small non zero eigenvalues of $\Delta_{f, h}^{(1)}$ and $m_{3}-b_{3}$ small non zero eigenvalues of $\Delta_{f, h}^{(2)}$.

2) The main obstacle to the complete analysis, for $n \geq 3$, is the construction of global quasimodes for general p-forms. At this time, we actually only know how to construct global quasimodes for functions, owing to the fact that $e^{-\frac{f}{h}}$ is in the kernel of $\Delta_{f, h}^{(0)}$. Such a construction means probably a deeper understanding of the Morse geometry than the one needed in [HeKlNi][HeNi][Lep1]. 3) Having in mind Lemma 4.2, one expects, in the general framework of $p$ forms in a n-dimensional space, to write the non zero eigenvalues of $\Delta_{f, h}^{(p)}$ on the form $A_{k}(h) e^{-2 \frac{\left|f\left(U_{i(k)}^{(p \pm 1)}\right)-f\left(U_{k}^{(p)}\right)\right|}{h}}\left(1+h c_{k}(h)\right)$, where $i$ is a one to one mapping from $\mathcal{U}^{(p)} \backslash\left\{U_{1}^{(p)}, \ldots, U_{b_{p}}^{(p)}\right\}$ into $\mathcal{U}^{(p-1)} \cup \mathcal{U}^{(p+1)}$; the points $U_{1}^{(p)}, \ldots, U_{b_{p}}^{(p)}$ being those associated with the $b_{p}$ zero eigenvalues of $\Delta_{f, h}^{(p)}$.

Acknowledgement: The author would like to thank T. Jecko, F. Nier and M. Klein for profitable discussions. 


\section{References}

[BoEcGaKl] A. Bovier, M. Eckhoff, V. Gayrard, and M. Klein: Metastability in reversible diffusion processes I: Sharp asymptotics for capacities and exit times. JEMS 6 (4), pp. 399-424 (2004).

[BoGaKl] A. Bovier, V. Gayrard, and M. Klein. Metastability in reversible diffusion processes II: Precise asymptotics for small eigenvalues. JEMS 7 (1), pp. 69-99 (2004).

[FrWe] M.I. Freidlin and A.D. Wentzell. Random perturbations of $d y$ namical systems. Transl. from the Russian by Joseph Szuecs. 2nd ed. Grundlehren der Mathematischen Wissenschaften, 260, New York (1998).

[Gra] A. Gramain. Cours d'initiation à la topologie algébrique et réelle, année 1969-1970. Publications mathématiques d'Orsay, Orsay : Service des publications, Faculté des sciences (1970).

[Hen] G. Henniart. Les inégalités de Morse (d'après E. Witten). Seminar Bourbaki, Vol. 1983/84, Astérisque No. 121-122, pp. 43-61 (1985) .

[HeKlNi] B. Helffer, M. Klein, and F. Nier. Quantitative analysis of metastability in reversible diffusion processes via a Witten complex approach. Matematica Contemporanea, 26, pp. 41-85 (2004).

[HeNi] B. Helffer and F. Nier. Quantitative analysis of metastability in reversible diffusion processes via a Witten complex approach: the case with boundary. Mémoire 105, Société Mathématique de France (2006).

[HeSj4] B. Helffer and J. Sjöstrand. Puits multiples en limite semi-classique IV -Etude du complexe de Witten -. Comm. Partial Differential Equations 10 (3), pp. 245-340 (1985).

[HoKuSt] R. Holley, S. Kusuoka, and D. Stroock. Asymptotics of the spectral gap with applications to the theory of simulated annealing. J. Funct. Anal. 83 (2), pp. 333-347 (1989).

[Kol] V.N. Kolokoltsov. Semi-classical analysis for diffusions and stochastic processes. Lecture Notes in Mathematics 1724, Springer Verlag (2000).

[Lep1] D. Le Peutrec. Small eigenvalues of the Neumann realization of the semiclassical Witten Laplacian. Preprint (2008). http://hal.archivesouvertes.fr/hal-00297207/fr/ 
[Mic] L. Miclo. Comportement de spectres d'opérateurs à basse température. Bull. Sci. Math. 119, pp. 529-533 (1995).

[Mil] J.W. Milnor. Morse Theory. Princeton University press (1963).

[Nie] F. Nier, Quantitative analysis of metastability in reversible diffusion processes via a Witten complex approach. Journées "Equations aux Dérivées Partielles", Exp No VIII, Ecole Polytechnique (2004).

[Wit] E. Witten. Supersymmetry and Morse inequalities. J. Diff. Geom. 17, pp. 661-692 (1982). 